\title{
A Fluorescence Assay for Exosome Detection Based on Bivalent Cholesterol Anchor Triggered Target Conversion and Enzyme-Free Signal Amplification
}

\author{
Xiaokun Wang, Hezhen Shang, Cuiping Ma,* and Lingxin Chen* \\ Cite This: Anal. Chem. 2021, 93, 8493-8500 \\ Read Online
}

ACCESS | Lill Metrics \& More | 回 Article Recommendations ｜ sl Supporting Information

ABSTRACT: Exosomes are emerging as one of the most promising biomarkers for early disease diagnosis and prognosis. The significant challenges facing the available methods include improving the detection specificity and sensitivity in complex biological samples. Herein, a fluorescence assay was established based on a combination of immunomagnetic separation and a twostep signal amplification strategy for direct isolation and subsequent detection of exosomes. First, immunomagnetic beads capture and enrich the exosomes via antibody-antigen reactions. Second, bivalent cholesterol (BC) anchors spontaneously insert into the lipid bilayer of bead-captured exosomes, forming a "one to many" amplification effect. The simultaneous recognition of the

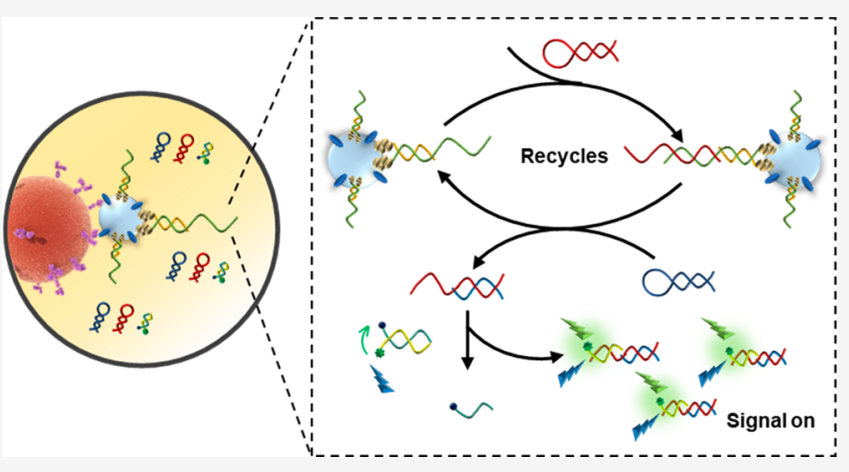
surface protein and the lipid bilayer structure of the exosome significantly eliminates the interference risk from free proteins. The detection of exosomes converts to the detection of $\mathrm{BC}$-anchors. Finally, the sticky end of the BC-anchor acts as the initiator to trigger the enzyme-free DNA circuits for secondary signal amplification. Under the optimal conditions, highly sensitive and selective detection of exosomes was achieved ranging from $5.5 \times$ $10^{3}$ to $1.1 \times 10^{7}$ particles $/ \mu \mathrm{L}$ with a limit of detection of $1.29 \times 10^{3}$ particles $/ \mu \mathrm{L}$. Moreover, this method allows the isolation and quantitative analysis of exosomes in several biological fluids with satisfactory recovery rates (92.25-106.8\%). Thus, this approach provides a sensitive, anti-interference platform for isolating and detecting exosomes.

$\mathrm{E}$ xosomes, a type of extracellular vesicle, are actively secreted by both normal and cancer cells. ${ }^{1-3}$ They carry various biological constituents of their parent cells, including nucleic acids, bioactive lipids, carbohydrates, and transmembrane and cytosolic proteins, which play essential roles in different biological processes (e.g., intercellular communication, inflammation processes, and tissue regeneration). ${ }^{4-7}$ Although their clinical application is still in its infancy, extensive evidence has demonstrated that tumor-derived exosomes can influence the recruitment and reprogramming of elements associated with the tumor microenvironment; their contents can also predict the origins of parental tumors. ${ }^{8,9}$ Tumor-derived exosomes express cancer-specific proteins and nucleic acids during carcinogenesis, and the total number of exosomes in cancer patients is significantly larger than that in healthy individuals. ${ }^{10,11}$ Besides, exosomes exist in almost all body fluids (blood, urine, saliva, cerebrospinal fluid, breast milk, et al.) with high abundance and stability, which are obtained easily and do not cause any trauma to the body. ${ }^{12,13}$ Therefore, exosomes become reliable and ideal noninvasive biomarkers for cancer diagnosis and prognosis.

As is well-known, biological fluids contain many complex interferents, such as cell debris, proteins, and other types of vesicles. Usually, isolation and enrichment steps are required before exosome analysis. Traditional isolation methods of exosomes typically include ultra-centrifugation (UC), coprecipitation, size-exclusion chromatography, etc. ${ }^{14,15}$ Among these, UC is the widely used gold standard for exosome isolation. However, it faces some limitations, such as the requirement of expensive equipment, skilled operators, large sample volumes, tedious steps, and lengthy processing. ${ }^{16}$ To date, various analytical methods, including nanoparticle tracking analysis (NTA), Western blot (WB), and enzyme-linked immunosorbent assay (ELISA), have been extensively used for the detection of exosomes. NTA is the most popular technique to quantify individual exosomes, but the quantification accuracy is easily interfered with by lipoproteins or protein aggregates, and it cannot selectively detect specific exosome subpopulations. ${ }^{17,18}$ ELISA and WB can differentiate cancer

Received: February 21, 2021

Accepted: June 7, 2021

Published: June 11, 2021 
exosomes from normal exosomes by recognizing their specific proteins. Nonetheless, they suffer from limitations in their "low sensitivity" or "limit in quantitative analysis". ${ }^{19-21}$ All the drawbacks limit their application in clinical diagnosis. Therefore, new methods for sensitive and straightforward exosome isolation and detection are still needed.

Recently, Zarovni et al. developed an immunomagnetic bead-based exosome isolation method via the binding specificity between typical exosome markers and their corresponding antibodies immobilized on magnetic beads. ${ }^{22}$ The use of the immunomagnetic beads greatly simplifies the isolation procedure; the impurities and excess reagents can be effectively removed by magnetic separation. Particularly, this immunomagnetic bead-based protocol not only allows the specific subpopulation of exosomes to be isolated but also has good compatibility with downstream detection, such as electrochemistry, ${ }^{23,24}$ colorimetry, ${ }^{25,26}$ fluorescence, ${ }^{27-30}$ and surface-enhanced Raman scattering techniques. ${ }^{31,32}$ Of these, fluorescence has attracted significant attention due to its extraordinary sensitivity and simplicity as well as many signal providers. However, the fluorescence assays for specially and accurately analyzing exosomes in complex biological samples are still challenging. Most of the reported fluorescence assays quantify exosomes by recognizing an exosome-specific protein (e.g., CD63, CD9, GPC-1). Unfortunately, undesired false signals would be triggered by free proteins from ruptured cells or exosomes. Therefore, simultaneous recognition of two or more exosome-specific sites is necessary to reduce free proteins' interference. Moreover, in the early stages of cancer, the detection is usually hampered by the low abundance of tumor exosomes; thus, signal amplification strategies are necessary for the fluorescence assays to improve the detection sensitivity. Signal amplification, including enzyme-assisted and enzyme-free amplification strategies, has become a popular and reliable method to improve detection sensitivity in the last decades. $^{33,34}$ Compared with the enzyme-assisted signal amplification strategy, the enzyme-free amplification strategy featured good stability, low cost, simplicity, and the exemption of complex enzymatic reactions. ${ }^{35,36}$

Herein, we developed a simple yet powerful fluorescence assay based on magnetic isolation and enzyme-free signal amplification to detect exosomes. This proposed method consists of three components. First, anti-CD63 antibodies immobilized MBs (anti-CD63 MBs) were employed to capture and enrich exosomes from complex samples. Second, BCanchors spontaneously insert into the lipid bilayer of exosomes captured on anti-CD63 MBs through the hydrophobic interaction between cholesterol moieties and the lipid layer, ${ }^{37,38}$ the trigger sequences remaining pointed outward. The simultaneous recognition of surface proteins and the lipid bilayer of exosomes means that exosome-specific protein or lipid membrane alone could not interfere with the demonstrated assay. Finally, the presence of hairpin structures and fluorescence reporters initiates the enzyme-free DNA circuits for signal amplification. It is noticeable that only the presence of exosomes could begin the following enzyme-free DNA circuits. We believe that this proposed fluorescence assay overcomes the technical limitations such as detection specificity and sensitivity in conventional methods.

\section{EXPERIMENTAL SECTION}

Materials. 4-Morpholineethanesulfonic acid (MES), bovine serum albumin (BSA), 1-ethyl-3-(3-(dimethylaminopropyl)- carodiimide (EDC), N-hydroxysuccinimide (NHS), sodium chloride $(\mathrm{NaCl})$, and potassium chloride $(\mathrm{KCl})$ were purchased from Sigma-Aldrich (St. Louis, MO). Carboxylicactivated magnetic beads were obtained from BioMag Scientific Inc. (Shanghai, China). Exosome CD63 and TSG101 protein detection kit were purchased from Umibio, Co., Ltd. (Shanghai, China). Anti-CD63 antibody, HPRlabeled secondary antibody, phosphate buffer solution (PBS), Tris-hydrochloride buffer (Tris-HCl), MES, and Tween 20 were purchased from BBI Life Sciences (Shanghai, China). Other reagents and all of the oligonucleotide sequences [Table S1, Supporting Information (SI)] used in this work were purchased from Sangon Biological Engineering Technology \& Services Co., Ltd. (Shanghai, China). Both $0.22 \mu \mathrm{m}$ filters and $30 \mathrm{kDa}$ ultra-centrifugal filters were purchased from Millipore Corp. (Billerica. MA). The ultrapure water $\left(18 \mathrm{M} \Omega \cdot \mathrm{cm}^{-1}\right)$ used in this work was obtained from a Milli-Q water purification system (Millipore Corp., MA). All reagents were of analytical reagent grade and used without further purification.

Before the experiment, BC-anchors (I and Ic mixed in 1:1 molar ratio) were annealed in PBS buffer by heating to $95{ }^{\circ} \mathrm{C}$ for $1 \mathrm{~min}$ followed by slowly decreasing the temperature to 23 ${ }^{\circ} \mathrm{C}$ within $13 \mathrm{~min}$. DNA hairpins 1 (H1), DNA hairpins 2 (H2), and RFQ probe were annealed in TNK buffer by the same method. RFQ probes are prepared by annealing FAMlabeled DNA sequence and Dabcyl-labeled DNA sequence at a ratio of 1 to 2 (FAM is the fluorescent molecule and Dabcyl is the quencher molecule).

Buffer solutions used in this work were as follows: $20 \mathrm{mM}$ Tris- $\mathrm{HCl}$ containing $150 \mathrm{mM} \mathrm{NaCl}$ and $0.1 \%$ Tween 20 (TBST); ultrapure water containing $40 \mathrm{mM}$ Tris, $20 \mathrm{mM}$ acetic acid, and $1 \mathrm{mM}$ EDTA (TAE); $10 \mathrm{mM}$ PBS containing $0.01 \%$ Tween-20 (PBST); $10 \mathrm{mM}$ PBS containing $0.01 \%$ Tween-20 and $0.01 \%$ BSA (PBSTB); and $20 \mathrm{mM}$ Tris- $\mathrm{HCl}$ containing $140 \mathrm{mM} \mathrm{NaCl}$ and $5 \mathrm{mM} \mathrm{KCl}$ (TNK).

Isolation, Quantification, and Characterization of Exosomes. Clinical samples were obtained from the Qingdao Chengyang People's Hospital and the Affiliated Hospital of Qingdao University. Informed consent for the use of the human serum was obtained from all of the donors. And this work was approved by the ethics committees from the institutions involved. The collected serum samples were centrifuged at $4{ }^{\circ} \mathrm{C}, 3000 \mathrm{~g}$ for $10 \mathrm{~min}$ to remove the cell debris, and the supernatant was further centrifuged at $4{ }^{\circ} \mathrm{C}$, $15000 \mathrm{~g}$ for $20 \mathrm{~min}$ to remove other impurities. Then, the supernatant was collected and sequentially ultracentrifugation twice at $120000 \mathrm{~g}$ for $70 \mathrm{~min}$. Finally, the exosome pellets were resuspended in PBS solution and filtered using a $0.22 \mu \mathrm{m}$ syringe filter. The morphology of exosomes was observed by TEM. The concentration of the exosome was measured using NTA. The Western blotting analysis was used to verify the extracted exosomes' proteins using TSG101 and CD63 primary antibody. These obtained exosomes with exact concentrations were further used as standard.

Preparation of Anti-CD63 MBs. The carboxyl-terminal groups on the magnetic beads were activated by $5 \mu \mathrm{L}$ of EDC $(0.1 \mathrm{M})$ and $5 \mu \mathrm{L}$ of NHS $(0.1 \mathrm{M})$ under gentle shaking at room temperature. After $30 \mathrm{~min}$, the mixture was separated by a magnet and washed twice with MES (15 mM, pH 6.0). Subsequently, the mixture was resuspended in MES $(15 \mathrm{mM}$, $\mathrm{pH}$ 6.0) and reacted with $10 \mu \mathrm{L}$ of anti-CD63 antibodies (1 $\mathrm{mg} / \mathrm{mL}$ ) overnight under gentle shaking. The unreacted 
Scheme 1. Schematic Illustration of the Proposed Method for the Quantitative Evaluation of Exosome Based on Magnetic Separation and Enzyme-Free Signal Amplification

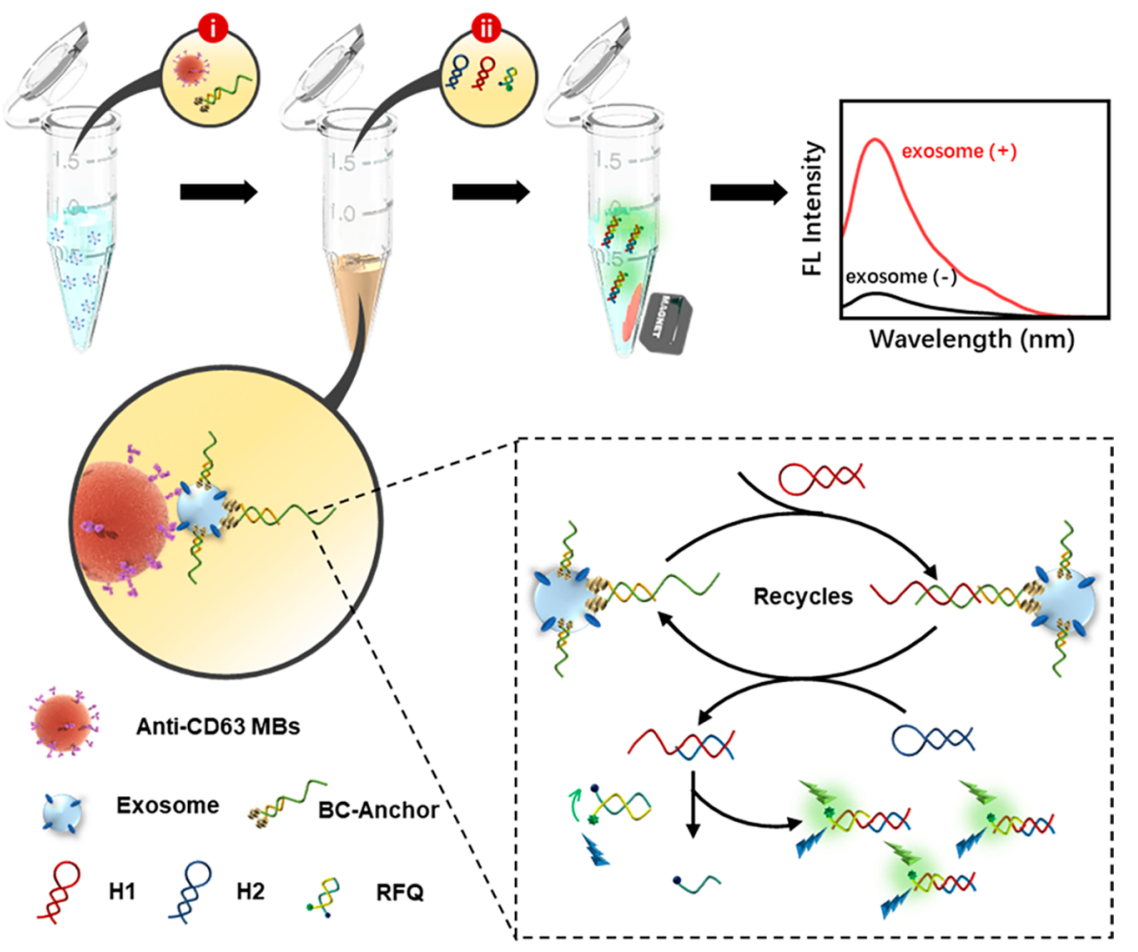

Scheme 2. Schematic Illustration of the Enzyme-Free DNA Circuitry for Signal Amplification

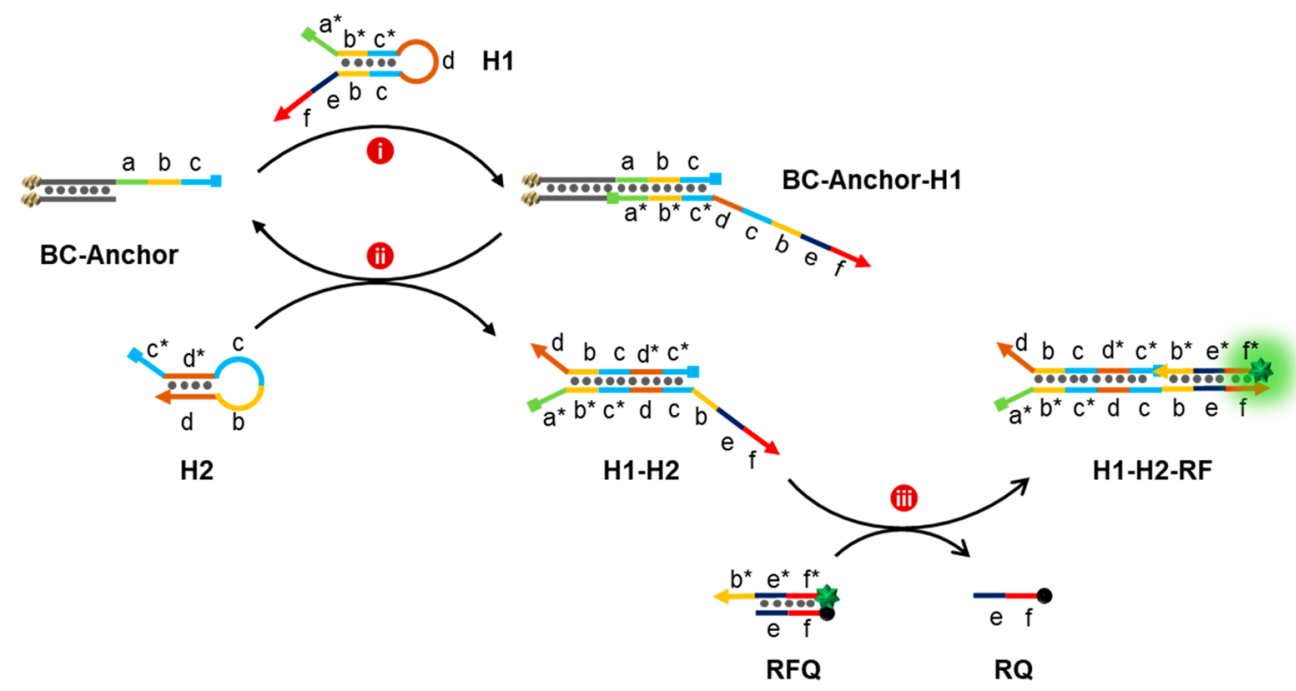

carboxyl terminal groups were deactivated with $20 \mu \mathrm{L}$ of BSA (2\%) for $30 \mathrm{~min}$. Finally, the mixture was washed three times with PBST (10 mM, pH 7.2), and the obtained anti-CD63 MBs were resuspended in PBSTB $(10 \mathrm{mM})$.

Exosome Quantification by the Fluorescence Assay. A $30 \mu \mathrm{L}$ portion of anti-CD63 MBs and $20 \mu \mathrm{L}$ of different concentrations of exosomes were mixed and incubated for $2 \mathrm{~h}$ with gentle shaking. The mixture was isolated by a magnetic separator and washed with PBST three times. Then, $50 \mu \mathrm{L}$ of $100 \mathrm{nM}$ BC-anchor was used to resuspend the above immunocomplexes, followed by incubation for $40 \mathrm{~min}$ at room temperature with gentle shaking. The immunocomplexes, now containing $\mathrm{BC}$-anchor inserted in the exosome lipid membrane, were isolated again by magnetic separation and were washed four times with PBST. A solution of $300 \mathrm{nM}$ $\mathrm{H} 1,450 \mathrm{nM} \mathrm{H} 2$, and $300 \mathrm{nM}$ RFQ was used to resuspend the above immunocomplexes for a $2 \mathrm{~h}$ incubation. Finally, the supernatant was measured at an excitation of $480 \mathrm{~nm}$. Besides, the proposed method's selectivity toward exosomes was investigated by replacing exosomes with other interferents to participate in the analysis strategy mentioned above.

\section{RESULTS AND DISCUSSION}

Principle of the Assay. An overview of the fluorescence assay for exosome detection is exhibited in Scheme 1. Immunomagnetic based capture and isolation approaches have the advantage of easy operation, high capture efficiency, and convenient integration and represent the most promising 

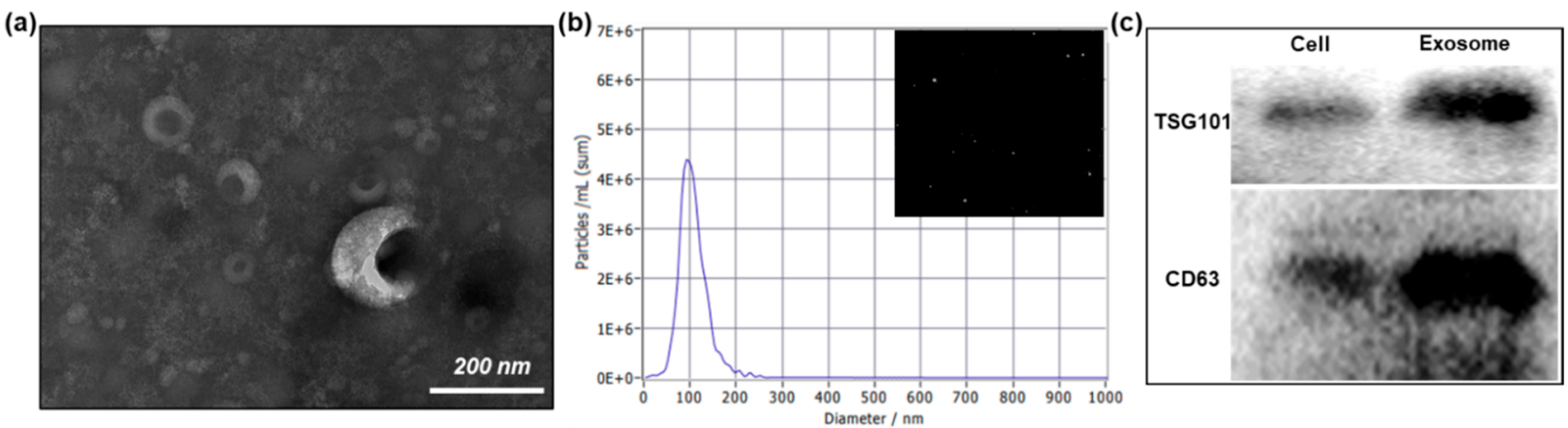

Figure 1. Characterization of exosomes: (a) TEM image of the extracted exosomes, (b) the size distribution of the extracted exosomes obtained by NTA, and (c) Western blot analysis of Hela cell lysates and the extracted exosomes.

technique for exosome isolation. ${ }^{22}$ As a proof-of-concept, we employed CD63, a well-known exosome biomarker expressed on the outer surface of exosomes, ${ }^{39,40}$ as a target model for immunoaffinity-based exosome isolation. Anti-CD63 antibodies were immobilized on carboxylic acid functionalized MBs through EDC/NHS coupling to fabricate the capture and isolation substrates. Two cholesterol-labeled DNA sequences with partially complementary regions, one of which has a sticky end, are designed to build the BC-anchors. When the exosomes-containing clinical sample was mixed with antiCD63 MBs in a microtube, exosomes were captured on the surface of anti-CD63 MBs through antibody-antigen reactions, and other interfering factors were washed away via magnetic separation. Subsequently, the BC-anchors were introduced and spontaneously inserted into the lipid bilayer membrane of exosomes captured on anti-CD63 MBs through the hydrophobic interaction between cholesterols and the lipid bilayer, the sticky end remaining pointed outward to trigger the enzyme-free DNA circuits.

In detail, the overall concept of the enzyme-free DNA circuits amplification process is shown in Scheme 2. Region a of the BC-anchor can function as a toehold to trigger interactions with the exposed region a of $\mathrm{H} 1$, initiating a branch migration reaction to open hairpin $\mathrm{H} 1$ and form a $\mathrm{BC}$ anchor-H1 intermediate (i). Next, the newly exposed region c of $\mathrm{H} 1$ is free to hybridize with the toehold region $\mathrm{c}^{*}$ of $\mathrm{H} 2$ and hybridize with $\mathrm{H} 2$, which leads to the dissociation of $\mathrm{BC}$ anchor from the $\mathrm{H} 1-\mathrm{H} 2$ duplex (ii). The released BC-anchor will be available for additional rounds of strand displacement, which produced numerous $\mathrm{H} 1-\mathrm{H} 2$ duplexes. The exposed toehold region of the $\mathrm{H} 1-\mathrm{H} 2$ duplex continues to hybridize with region $b^{*}$ of RFQ. It triggers the branch migration reaction to displace the RQ quencher, which restores the RF fluorescence signal in the obtained $\mathrm{H} 1-\mathrm{H} 2-\mathrm{RF}$ complex (iii). The fluorescence intensity change is proportional to the captured exosomes' concentration, allowing the exosomes' quantitative detection. However, in the absence of $\mathrm{BC}$-anchorlabeled exosomes, $\mathrm{H} 1$ and $\mathrm{H} 2$ cannot spontaneously hybridize with each other due to the effective block created by intramolecular hairpin structures. Annealing RF and RQ prepared RFQ duplexes at a ratio of 1 to 2 . An excess of RQ ensures efficient quenching of RF but does not interfere with the folded hairpin structures. ${ }^{35,36}$ The developed method is expected to possess high sensitivity due to antibody recognition and the use of the cholesterol-lipid membrane portion of the assay chemistry.

Characterization of Exosomes. Exosomes isolated by the ultracentrifugation method were used as the model to investigate the detection specificity and quantitative analysis capability of the proposed method. The morphology and concentration of the extracted exosomes were characterized by TEM and NTA. As shown in Figure 1a, the extracted exosomes have a typical cup-shaped membrane structure with a size ranging from 30 to $150 \mathrm{~nm}$. The NTA result shows that the extracted exosomes' size distribution varied from 30 to 230 $\mathrm{nm}$, a peak value with an average diameter centered at $97 \mathrm{~nm}$ (Figure 1b). The morphology and size characteristics are in good accordance with the previous literature. ${ }^{1,41}$ Additionally, the useful exosome markers CD63 and TSG 101 were validated by Western blotting analysis. A Hela cell lysates standard sample from a commercial protein detection kit was prepared as a positive control. As shown in Figure 1c, clear bands of TSG 101 and CD63 were observed on the gel, which confirmed the abundance of CD63 on the extracted exosomes. All the results verified that we have successfully extracted and purified serum exosomes.

Feasibility of the Working Principle. The enzyme-free DNA circuitry is of great importance for signal amplification. Here, PAGE analysis was employed to verify the feasibility of DNA circuits triggered by BC-anchors. As shown in Figure 2a, lanes 2, 3, and 4 display $\mathrm{H} 1, \mathrm{H} 2$, and RFQ bands, respectively. Lane 5 displays the bands of $\mathrm{H} 1$ and $\mathrm{H} 2$, which demonstrates that $\mathrm{H} 1$ and $\mathrm{H} 2$ could coexist stably due to being kinetically hindered. While in the presence of $\mathrm{BC}$-anchors, a new band of $\mathrm{H} 1-\mathrm{H} 2$ duplexes was observed, and the $\mathrm{H} 1, \mathrm{H} 2$ bands significantly reduced in lane 6 . Nevertheless, in the presence of RFQ probes, the band of $\mathrm{H} 1-\mathrm{H} 2$ duplexes disappeared, and a new band of $\mathrm{H} 1-\mathrm{H} 2-\mathrm{RF}$ complex appeared, which demonstrated the release of RF from RFQ probes. Additionally, the amplification process was also verified by fluorescence analysis. As shown in Figure 2b, in the presence of BC-anchors, the amplification reaction yielded a significant fluorescence signal. However, in the absence of BC-anchors, nearly no fluorescence signal was observed. The results above corroborated that the amplification reaction successfully happened with low background, and numerous $\mathrm{H} 1-\mathrm{H} 2-\mathrm{RF}$ complexes were generated.

As mentioned before, carboxylic acid functionalized MBs were employed for the fabrication of capture and isolation substrates. Anti-CD63 antibodies were immobilized on the surfaces of carboxylic acid functionalized MBs through EDC/ NHS coupling. BSAs blocked the remaining activated sites of MBs to prevent nonspecific binding. To identify the conjugation, anti-mouse IgG-HRP was incubated with bare and anti-CD63 MBs, respectively. After washing via magnetic separation, TMB and TMB stop solution were sequentially 

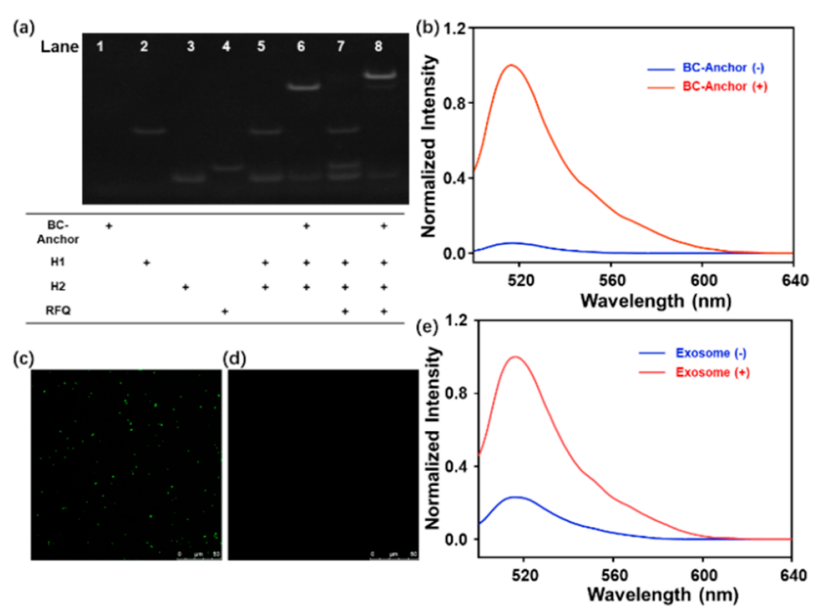

Figure 2. (a) PAGE electrophoresis image of different samples in the amplification assay: lane 1, $5 \mathrm{nM}$ of BC-anchor; lane 2, $90 \mathrm{nM}$ of $\mathrm{H1}$; lane 3, $135 \mathrm{nM}$ of $\mathrm{H2}$; lane 4, $90 \mathrm{nM}$ of RFQ; lane 5, $90 \mathrm{nM}$ of $\mathrm{H} 1$ and $135 \mathrm{nM}$ of $\mathrm{H} 2$; lane 6, $5 \mathrm{nM}$ of BC-anchor, $90 \mathrm{nM}$ of $\mathrm{H} 1$, and 135 $\mathrm{nM}$ of $\mathrm{H} 2$; lane 7, $90 \mathrm{nM}$ of $\mathrm{H} 1,135 \mathrm{nM}$ of $\mathrm{H} 2$, and $90 \mathrm{nM}$ of RFQ; lane $8,5 \mathrm{nM}$ of BC-anchor, $90 \mathrm{nM}$ of $\mathrm{H1}, 135 \mathrm{nM}$ of $\mathrm{H} 2$, and $90 \mathrm{nM}$ of RFQ. The mixtures were incubated at room temperature for $2 \mathrm{~h}$. (b) Fluorescence spectra corresponding to lanes 7 and 8. Confocal microscopy fluorescence images after binding anti-CD63 MBscaptured exosomes (c) and anti-CD63 MBs (d) with BC-anchorAlexa Fluor 488 complexes. (e) Fluorescence spectra of the reaction system with and without exosome.

added (time delay $\sim 15 \mathrm{~min}$ ). It is well-known that HRP usually catalyzes the oxidation of TMB, which produces a blue reaction product. The color then changes to yellow with the TMB stop solution with a maximum absorbance at $450 \mathrm{~nm}$. As shown in Figure S1 (SI), the color change and absorbance at $450 \mathrm{~nm}$ for bare and anti-CD63 MBs indicated that anti-CD63 antibodies were successfully adsorbed on MBs' surfaces.

As one of the critical elements of this method, the insertion of $\mathrm{BC}$-anchors into the lipid bilayer of the exosomes was also investigated by using fluorescence microscopy. We designed an Alexa Fluor 488 labeled DNA sequence to hybridize with the sticky end of $\mathrm{BC}$-anchors. As shown in Figure 2c, in the presence of exosomes, the BC-anchor-Alexa Fluor 488 complexes spontaneously inserted into the lipid bilayer membrane and a bright fluorescence surrounding the MBs was observed. However, nearly no fluorescence was observed without the addition of exosomes (Figure $2 \mathrm{~d}$ ). The results suggested that $\mathrm{BC}$-anchors could successfully insert into the lipid bilayer membrane of exosome with low nonspecific binding with anti-CD63 MBs. Furthermore, Figure 2e shows the fluorescence response with or without the addition of exosomes. As expected, the high fluorescence signal was observed with exosomes' addition after insertion of BCanchors and subsequent enzyme-free amplification, but a quite weak fluorescence signal (the background signal) was observed in the absence of exosomes. Therefore, all of the above results revealed the feasibility of exosome detection with the proposed fluorescence assay based on magnetic separation and enzymefree signal amplification.

Optimization of the Experimental Conditions. To determine the optimal assay conditions, several experimental parameters were systematically optimized, including anchor structure, concentration ratios of $\mathrm{H} 1$ to $\mathrm{H} 2$, and amplification time. Optimization was operated by varying one parameter while others were kept constant.
First, we studied the effect of the anchor structure because the insertion of trigger sequences into the exosomes can influence this strategy's sensitivity. The monovalent cholesterol (MC) anchor was a single cholesterol tagged trigger sequence. The $\mathrm{BC}$-anchor was constructed by prehybridizing I and Ic with 15 bp complementary regions and labeling cholesterol at the $3^{\prime}$ and $5^{\prime}$ terminals. As shown in Figure 3, the BC-anchor
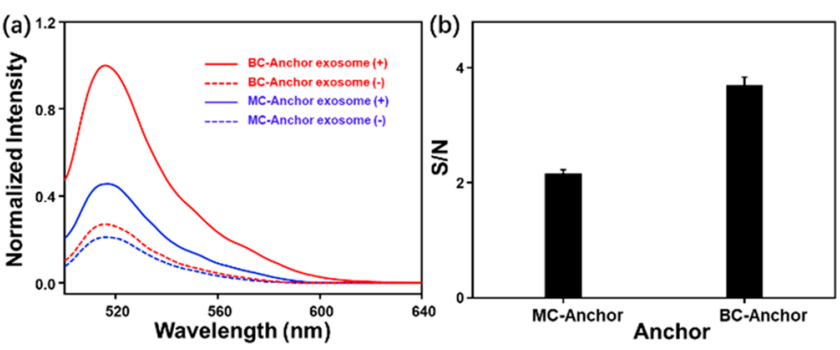

Figure 3. Optimization of the anchor structure in the assay. (a) The fluorescence spectra of the assay with MC-anchor (blue line) and BCanchor (red line) in the absence (dashed line) and presence (solid line) of exosomes. (b) $\mathrm{S} / \mathrm{N}$ ratio corresponding to panel a.

showed a higher signal-to-noise $(\mathrm{S} / \mathrm{N})$ ratio than the $\mathrm{MC}$ anchor, this is due to the complementary regions forcing the two cholesterol into proximity, providing a stronger binding force. ${ }^{37}$ The single-cholesterol-based anchoring of the trigger is weak; thus, $\mathrm{BC}$-anchor was used in the following assay.

Second, the probes $\mathrm{H} 1$ and $\mathrm{H} 2$ have a significant effect on the amplification efficiency. Sufficient amounts of $\mathrm{H} 2$ would guarantee the $\mathrm{BC}$-anchors' cyclic replacement from $\mathrm{BC}$ anchor-H1 complexes, but excess $\mathrm{H} 2$ could spontaneously hybridize with $\mathrm{H} 1$, generating background signals. Hence, five different concentration ratios of $\mathrm{H} 1$ to $\mathrm{H} 2$ were tested to determine which gave the best $\mathrm{S} / \mathrm{N}$ ratio. As shown in Figure $\mathrm{S} 2 \mathrm{a}(\mathrm{SI})$, the $\mathrm{S} / \mathrm{N}$ ratio reached the maximum when the concentration ratio of $\mathrm{H} 1$ to $\mathrm{H} 2$ was 1 :1.5. On the basis of this result, the concentration ratio of $1: 1.5$ was used in the following assays.

Additionally, the amplification time is another major factor influencing the amplification efficiency; too short an amplification time cannot produce sufficient $\mathrm{H} 1-\mathrm{H} 2-\mathrm{RF}$ complexes. Here, different amplification times ranging from 30 to $180 \mathrm{~min}$ were investigated. Figure S2b (SI) displays that the $\mathrm{S} / \mathrm{N}$ ratio increased with amplification time in the range from 30 to $120 \mathrm{~min}$ and then reached a balance beyond $120 \mathrm{~min}$. Thus, $120 \mathrm{~min}$ was long enough for the signal amplification process, and a further increase in the reaction time did not improve the amplification performance.

Analytical Performance of the Method. Under the optimized conditions, various concentrations of exosomes were used to evaluate the sensitivity and linear range of the proposed method. Figure 4a shows the fluorescence spectra for various concentrations of exosomes $\left(0,5.5 \times 10^{3}, 1.1 \times 10^{4}\right.$, $5.5 \times 10^{4}, 1.1 \times 10^{5}, 5.5 \times 10^{5}, 1.1 \times 10^{6}, 5.5 \times 10^{6}$, and $1.1 \times$ $10^{7}$ particle $\left./ \mu \mathrm{L}\right)$. In the absence of exosomes, a weak fluorescence signal was observed. As expected, the fluorescence signals were regularly enhanced with increasing the concentration of exosomes. Figure $4 \mathrm{~b}$ displays the calibration curve of the fluorescent ratios $\left(F / F_{0}\right)$ as a function of the concentrations of the exosome, where $F$ and $F_{0}$ are respectively the fluorescence intensity with and without the addition of exosomes. The inset displays the linear relationship between the fluorescence intensity ratio $\left(F / F_{0}\right)$ and logarithmic values 


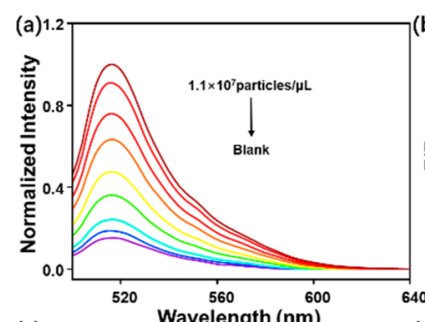

(c)
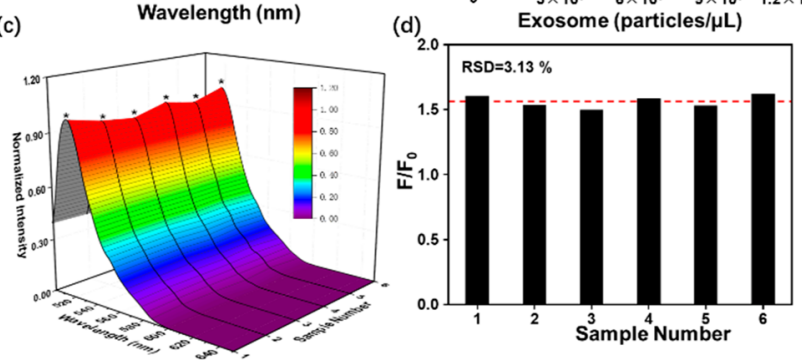

Figure 4. (a) Normalized fluorescence spectra response to different concentrations of exosomes. (b) Calibration curve of the fluorescent ratios $\left(F / F_{0}\right)$ as a function of the concentrations of the exosome. The inset shows the linear relationship between the fluorescence intensity ratio $\left(F / F_{0}\right)$ and logarithmic values of exosome concentrations. The error bars indicate the standard deviations calculated from three measurements. Reproducibility test of the proposed method: (c) normalized fluorescence spectra and (d) corresponding intensity for $1.1 \times 10^{4}$ particle $/ \mu \mathrm{L}$ of the exosome. The relative standard deviation (RSD) for the six independent tests was calculated to be 3.13\%.

of exosome concentrations (from $5.5 \times 10^{3}$ to $1.1 \times 10^{7}$ particle $/ \mu \mathrm{L}$ ) with a corresponding correlation coefficient $\left(R^{2}\right)$ of 0.992 . The LOD value was estimated to be $1.29 \times 10^{3}$ particle $/ \mu \mathrm{L}$ based on $3 \sigma_{\mathrm{b}} / \mathrm{s}\left(\sigma_{\mathrm{b}}\right.$ is the standard deviation of the blank samples, and $s$ is the slope of the linear calibration). These results confirmed that the proposed method could be used for the quantitative analysis of exosomes.

Reproducibility is a vitally important factor for quantitative assays. To evaluate the reproducibility of the proposed method, six repeated tests were performed for the same concentration of exosome $\left(1.1 \times 10^{4}\right.$ particle $\left./ \mu \mathrm{L}\right)$. The corresponding fluorescence spectra are displayed in Figure 4c. On the basis of this result, the RSD was estimated to be $3.13 \%$ (Figure $4 \mathrm{~d}$ ), which confirmed the good reproducibility of the proposed method.

Its analytical performance was also compared with the MBsintegrated ELISA method. As shown in Figure S3 (SI), the LOD determined by ELISA was estimated to be $5.09 \times 10^{5}$ particle $/ \mu \mathrm{L}$. On the basis of this result, it is concluded that the value determined by the proposed assay is approximately 2 orders of magnitude more sensitive than that obtained from ELISA. Meanwhile, the results were also compared with the previously reported works using other detection techniques. As shown in Table S2 (SI), the LOD of the proposed method is not better than the electrochemical methods (70 and 96 particle $/ \mu \mathrm{L})$. However, it exhibited excellent sensitivity compared with various currently available methods, such as colorimetric assay $\left(5.2 \times 10^{5}\right.$ particle $\left./ \mu \mathrm{L}\right)$, lateral flow assay $\left(1.4 \times 10^{4}\right.$ particle $\left./ \mu \mathrm{L}\right)$, and fluorescence assay $\left(4.8 \times 10^{4}\right.$ particle $/ \mu \mathrm{L})$. The great sensitivity was mainly due to the specific recognition between exosomes and their corresponding antibodies and the efficient signal amplification strategy. Additionally, the simultaneous recognition of exosome surface protein CD63 and the lipid bilayer structure will greatly reduce the interference risk from free proteins (Figure S4, SI).
Detection of Exosomes in Complex Biological Samples. We next investigated the anti-interference ability of the proposed method. Different actual samples including $20 \%$ exosomes-depleted fetal bovine serum (ED-FBS), 20\% ultracentrifuged serum (UC-serum), and $20 \%$ ultracentrifuged urine (UC-urine) were spiked for recovery experiments. UCserum and UC-urine samples were prepared by centrifuging at $4{ }^{\circ} \mathrm{C}, 120000 \mathrm{~g}$ for $24 \mathrm{~h}$ to remove the exosomes. Then, the supernatant was collected and filtered using a $30 \mathrm{kDa}$ ultracentrifugal filter. As shown in Table S3 (SI), satisfactory recovery (92.25-106.8\%) and RSD (2.84-5.40\%) values were obtained, which indicated that the complex biological matrix has a negligible effect on our proposed method. This is mainly due to the superior separation capability of MBs. Besides, to further evaluate the proposed method's clinical diagnostic efficacy, freshly collected serum samples of healthy individuals and cancer patients (information is shown in Table S4, SI) were analyzed by using this method. As shown in Figure 5, the

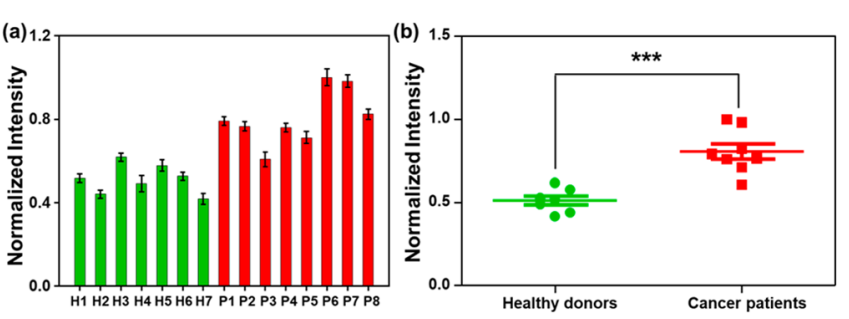

Figure 5. (a) Fluorescence intensity of clinical samples (seven healthy individuals and eight cancer patients) analyzed by the proposed method. (b) Scatter plot corresponding to panel a.

fluorescence intensity obtained from cancer samples and healthy samples are significantly statistically different $(P=$ $0.0002)$, consistent with previous reports that there is increased secretion of exosomes in cancer patients compared to healthy individuals. But more clinical samples are needed to validate this phenomenon in the field of clinical diagnosis.

\section{CONCLUSION}

In the present study, we report an efficient and accurate method for selectively isolating and quantifying exosomes from complex biological samples based on magnetic separation and a two-step signal amplification strategy. The proposed method has the following advantages. (i) The use of anti-CD63 MBs and $\mathrm{BC}$-anchors simplifies the isolation process and can eliminate the interference from free proteins. (ii) The insertion of $\mathrm{BC}$-anchors generates the "one exosome to numerous $\mathrm{BC}$ anchors" signal amplification effect. (iii) An enzyme-free signal amplification strategy avoids natural enzymes' use, reducing the cost and operating and storage facilities usually required for natural enzymes. The fluorescence intensity of the supernatant can indirectly estimate the concentration of the exosomes.

The LODs of exosomes determined from the proposed fluorescence assay were estimated to be $1.29 \times 10^{3}$ particles/ $\mu \mathrm{L}$; these results indicated approximately 2 orders of magnitude more sensitivity than the MBs-integrated ELISA method. Meanwhile, this method successfully isolated and detected exosomes from spiked biological samples (e.g., FBS, serum, and urine) with recovery values ranging from $92.25 \%$ to $106.8 \%$. This method can also measure the difference in exosome concentration between serums of healthy individuals and cancer patients. We believe that this proposed fluorescence 
assay will provide a new platform for accurate and specific detection of exosomes in complex biological samples.

\section{ASSOCIATED CONTENT}

\section{SI Supporting Information}

The Supporting Information is available free of charge at https://pubs.acs.org/doi/10.1021/acs.analchem.1c00796.

General experimental section, sequences of oligonucleotides used in this work, optimization of experimental conditions, absorption result response to different concentrations of exosomes, evaluation of assay specificity, comparison among exosome detection methods, recovery values of exosomes spiked into real samples, and clinical information of healthy donors and cancer patients (PDF)

\section{AUTHOR INFORMATION}

\section{Corresponding Authors}

Cuiping Ma - Shandong Provincial Key Laboratory of Biochemical Engineering, College of Marine Science and Biological Engineering, Qingdao University of Science and Technology, Qingdao 266042, China; Email: mcp169@ 163.com

Lingxin Chen - CAS Key Laboratory of Coastal Environmental Processes and Ecological Remediation, Shandong Key Laboratory of Coastal Environmental Processes, Yantai Institute of Coastal Zone Research, Chinese Academy of Sciences, Yantai 264003, China; The Key Laboratory of Life-Organic Analysis, Key Laboratory of Pharmaceutical Intermediates and Analysis of Natural Medicine, College of Chemistry and Chemical Engineering, Qufu Normal University, Qufu 273165, China; School of Pharmacy, Binzhou Medical University, Yantai 264003, China; (1) orcid.org/0000-0002-3764-3515; Email: lxchen@yic.ac.cn

\section{Authors}

Xiaokun Wang - Shandong Provincial Key Laboratory of Biochemical Engineering, College of Marine Science and Biological Engineering, Qingdao University of Science and Technology, Qingdao 266042, China

Hezhen Shang - Department of Hepatobiliary Surgery, Qingdao Chengyang District People's Hospital, Qingdao 266109, China

Complete contact information is available at:

https://pubs.acs.org/10.1021/acs.analchem.1c00796

\section{Notes}

The authors declare no competing financial interest.

\section{ACKNOWLEDGMENTS}

We thank the National Nature Science Foundation of China (Nos. 21904078, 21976209), Taishan Scholar Project Special Funding (No. ts20190962), and the Key Project of Natural Science Foundation of Shandong Province (ZR2020KH030).

\section{REFERENCES}

(1) Théry, C.; Zitvogel, L.; Amigorena, S. Nat. Rev. Immunol. 2002, $2,569-579$.

(2) Mathieu, M.; Martin-Jaular, L.; Lavieu, G.; Théry, C. Nat. Cell Biol. 2019, 21, 9-17.
(3) Trajkovic, K.; Hsu, C.; Chiantia, S.; Rajendran, L.; Wenzel, D.; Wieland, F.; Schwille, P.; Brügger, B.; Simons, M. Science 2008, 319, 1244-1247.

(4) Jeppesen, D. K.; Fenix, A. M.; Franklin, J. L.; Higginbotham, J. N.; Zhang, Q.; Zimmerman, L. J.; Liebler, D. C.; Ping, J.; Liu, Q.; Evans, R.; Fissell, W. H.; Patton, J. G.; Rome, L. H.; Burnette, D. T.; Coffey, R. J. Cell 2019, 177, 428-445.

(5) Sung, B. H.; Ketova, T.; Hoshino, D.; Zijlstra, A.; Weaver, A. M. Nat. Commun. 2015, 6, 7164.

(6) Takasugi, M.; Okada, R.; Takahashi, A.; Virya Chen, D.; Watanabe, S.; Hara, E. Nat. Commun. 2017, 8, 15729.

(7) Azmi, A. S.; Bao, B.; Sarkar, F. H. Cancer Metastasis Rev. 2013, 32, 623-642.

(8) Tran, P. H. L.; Xiang, D.; Tran, T. T. D.; Yin, W.; Zhang, Y.; Kong, L.; Chen, K.; Sun, M.; Li, Y.; Hou, Y.; Zhu, Y.; Duan, W. Adv. Mater. 2020, 32, 1904040.

(9) Park, J. E.; Tan, H. S.; Datta, A.; Lai, R. C.; Zhang, H.; Meng, W.; Lim, S. K.; Sze, S. K. Mol. Cell. Proteomics 2010, 9, 1085-1099.

(10) Liu, C.; Zhao, J.; Tian, F.; Cai, L.; Zhang, W.; Feng, Q.; Chang, J.; Wan, F.; Yang, Y.; Dai, B.; Cong, Y.; Ding, B.; Sun, J.; Tan, W. Nat. Biomed. Eng. 2019, 3, 183-193.

(11) Melo, S. A.; Sugimoto, H.; O'Connell, J. T.; Kato, N.; Villanueva, A.; Vidal, A.; Qiu, L.; Vitkin, E.; Perelman, L. T.; Melo, C. A.; Lucci, A.; Ivan, C.; Calin, G. A.; Kalluri, R. Cancer Cell 2014, 26, $707-721$.

(12) Pluchino, S.; Smith, J. A. Cell 2019, 177, 225-227.

(13) Melo, S. A.; Luecke, L. B.; Kahlert, C.; Fernandez, A. F.; Gammon, S. T.; Kaye, J.; LeBleu, V. S.; Mittendorf, E. A.; Weitz, J.; Rahbari, N.; Reissfelder, C.; Pilarsky, C.; Fraga, M. F.; PiwnicaWorms, D.; Kalluri, R. Nature 2015, 523, 177-182.

(14) Li, P.; Kaslan, M.; Lee, S. H.; Yao, J.; Gao, Z. Theranostics 2017, 7, 789-804.

(15) Yang, D.; Zhang, W.; Zhang, H.; Zhang, F.; Chen, L.; Ma, L.; Larcher, L. M.; Chen, S.; Liu, N.; Zhao, Q.; et al. Theranostics 2020, 10, 3684-3707.

(16) Konoshenko, M. Y.; Lekchnov, E. A.; Vlassov, A. V.; Laktionov, P. P. BioMed Res. Int. 2018, 2018, 8545347.

(17) Dragovic, R. A.; Gardiner, C.; Brooks, A. S.; Tannetta, D. S.; Ferguson, D. J.; Hole, P.; Carr, B.; Redman, C. W.; Harris, A. L.; Dobson, P. J.; et al. Nanomedicine 2011, 7, 780-788.

(18) Soo, C. Y.; Song, Y.; Zheng, Y.; Campbell, E. C.; Riches, A. C.; Gunn-Moore, F.; Powis, S. J. Immunology 2012, 136, 192-197.

(19) Lobb, R. J.; Becker, M.; Wen Wen, S.; Wong, C. S. F.; Wiegmans, A. P.; Leimgruber, A.; Möller, A. J. Extracell. Vesicles 2015, 4, 27031.

(20) Logozzi, M.; De Milito, A.; Lugini, L.; Borghi, M.; Calabro, L.; Spada, M.; Perdicchio, M.; Marino, M. L.; Federici, C.; Iessi, E.; et al. PLoS One 2009, 4, No. e5219.

(21) Yoshioka, Y.; Kosaka, N.; Konishi, Y.; Ohta, H.; Okamoto, H.; Sonoda, H.; Nonaka, R.; Yamamoto, H.; Ishii, H.; Mori, M.; Furuta, K.; Nakajima, T.; Hayashi, H.; Sugisaki, H.; Higashimoto, H.; Kato, T.; Takeshita, F.; Ochiya, T. Nat. Commun. 2014, 5, 3591.

(22) Zarovni, N.; Corrado, A.; Guazzi, P.; Zocco, D.; Lari, E.; Radano, G.; Muhhina, J.; Fondelli, C.; Gavrilova, J.; Chiesi, A. Methods 2015, 87, 46-58.

(23) Boriachek, K.; Islam, M. N.; Gopalan, V.; Lam, A. K.; Nguyen, N.-T.; Shiddiky, M. J. Analyst 2017, 142, 2211-2219.

(24) An, Y.; Li, R.; Zhang, F.; He, P. Anal. Chem. 2020, 92, 54045410.

(25) Zhang, Y.; Wang, D.; Yue, S.; Lu, Y.; Yang, C.; Fang, J.; Xu, Z. ACS sensors 2019, 4, 3210-3218.

(26) Zhang, Y.; Jiao, J.; Wei, Y.; Wang, D.; Yang, C.; Xu, Z. Anal. Chem. 2020, 92, 15244-15252.

(27) He, F.; Wang, J.; Yin, B.-C.; Ye, B.-C. Anal. Chem. 2018, 90, $8072-8079$.

(28) Yu, X.; He, L.; Pentok, M.; Yang, H.; Yang, Y.; Li, Z.; He, N.; Deng, Y.; Li, S.; Liu, T.; et al. Nanoscale 2019, 11, 15589-15595.

(29) Wu, M.; Chen, Z.; Xie, Q.; Xiao, B.; Zhou, G.; Chen, G.; Bian, Z. Biosens. Bioelectron. 2021, 171, 112733. 
(30) Zhao, X.; Luo, C.; Mei, Q.; Zhang, H.; Zhang, W.; Su, D.; Fu, W.; Luo, Y. Anal. Chem. 2020, 92, 5411-5418.

(31) Tian, Y.-F.; Ning, C.-F.; He, F.; Yin, B.-C.; Ye, B.-C. Analyst 2018, 143, 4915-4922.

(32) Pang, Y.; Shi, J.; Yang, X.; Wang, C.; Sun, Z.; Xiao, R. Biosens. Bioelectron. 2020, 148, 111800.

(33) Willner, I.; Shlyahovsky, B.; Zayats, M.; Willner, B. Chem. Soc. Rev. 2008, 37, 1153-1165.

(34) Zhang, D. Y.; Seelig, G. Nat. Chem. 2011, 3, 103-113.

(35) Li, B.; Ellington, A. D.; Chen, X. Nucleic Acids Res. 2011, 39, No. e110.

(36) Arter, W. E.; Yusim, Y.; Peter, Q.; Taylor, C. G.; Klenerman, D.; Keyser, U. F.; Knowles, T. P. ACS Nano 2020, 14, 5763-5771.

(37) Pfeiffer, I.; Höök, F. J. Am. Chem. Soc. 2004, 126, 1022410225 .

(38) Ercole, F.; Whittaker, M. R.; Quinn, J. F.; Davis, T. P. Biomacromolecules 2015, 16, 1886-1914.

(39) Kowal, J.; Arras, G.; Colombo, M.; Jouve, M.; Morath, J. P.; Primdal-Bengtson, B.; Dingli, F.; Loew, D.; Tkach, M.; Théry, C. Proc. Natl. Acad. Sci. U. S. A. 2016, 113, E968-E977.

(40) Raposo, G.; Stoorvogel, W. J. Cell Biol. 2013, 200, 373-383.

(41) Vlassov, A. V.; Magdaleno, S.; Setterquist, R.; Conrad, R. Biochim. Biophys. Acta, Gen. Subj. 2012, 1820, 940-948. 